\title{
Annual report of thoracic surgery service at Shanghai Chest Hospital in 2019
}

\author{
Yiyang Wang ${ }^{1}$, Zhitao Gu ${ }^{1}$, Feng Yao ${ }^{1}$, Teng Mao ${ }^{1}$, Rui Wang ${ }^{1}$, Yifeng Sun ${ }^{1}$, Zhigang Li $^{1}$, Jun Yang ${ }^{1}$, \\ Qiang Tan', Qingquan Luo', Wentao Fang ${ }^{1}$ \\ ${ }^{1}$ Department of Thoracic Surgery, Shanghai Chest Hospital, Jiaotong University Medical School, Shanghai, China; ${ }^{2}$ Department of Oncological \\ Surgery, Shanghai Chest Hospital, Jiaotong University Medical School, Shanghai, China \\ Correspondence to: Wentao Fang. Department of Thoracic Surgery, Shanghai Chest Hospital, Jiaotong University Medical School, Shanghai, China. \\ Email: vwtfang@hotmail.com.
}

\begin{abstract}
Background: The thoracic surgery team at the Shanghai Chest Hospital has been publishing its annual report since 2018, summarizing the services provided and major progress achieved over the previous year.

Methods: All patients receiving thoracic surgery service at the Department of Thoracic Surgery and the Department of Oncological Surgery of Shanghai Chest Hospital in 2019 were enrolled. The number of surgical resections, types of surgical procedures, histological types of the diseases, and perioperative outcomes were collected and compared with the results from previous years.

Results: In 2019, a total of 17,021 patients received thoracic surgeries at the Shanghai Chest Hospital, an increase of $21.1 \%$ over the previous year. These included 14,591 pulmonary resections, 1,017 esophageal procedures, 1,089 mediastinal procedures, 59 tracheal procedures, and 9 lung transplantations. The percentage of minimally invasive surgeries among all procedures was $89.8 \%$, with 714 single-institution robotic-assisted thoracic surgeries being performed. Inspiringly, the average length of hospital stay of patients undergoing pulmonary surgery, esophageal surgery, and mediastinal surgery has continually decreased over the past 6 years. Meanwhile, the rate of major complications has remained low, with the inhospital mortality rate being only $0.18 \%$ in 2019 .

Conclusions: The services provided and progress made in 2019 by the thoracic surgery team of the Shanghai Chest Hospital were reviewed in this annual report, which is a reflection of our constant effort to help our patients with high-standard service and state-of-the-art techniques.
\end{abstract}

Keywords: Shanghai Chest Hospital; thoracic surgery; 2019

Received: 15 December 2020; Accepted: 07 January 2021; Published: 10 April 2021.

doi: $10.21037 /$ shc-2021-01

View this article at: http://dx.doi.org/10.21037/shc-2021-01

\section{Introduction}

In running one of the largest thoracic surgery centers in China, the thoracic surgery team of the Shanghai Chest Hospital has exerted continuous effort in the surgical treatment of thoracic diseases. In 2019, a total 17,021 patients received thoracic surgeries at the hospital, including 14,591 pulmonary resections, 1,017 esophageal procedures, 1,089 mediastinal procedures, 59 tracheal procedures, and 9 lung transplantations. The total case volume increased by $21.1 \%$ compared to that of the year before, while the percentage of minimally invasive surgery (MIS) was as high as $89.8 \%$, and included both videoassisted thoracic surgery (VATS) and robotic-assisted thoracic surgery (RATS) (Figure 1). The indication for minimally invasive surgery was no longer reserved solely for early-stage thoracic diseases, but was extended to those patients with locally advanced tumors, those who had received neoadjuvant therapy, and those needing complex reconstructions after extensive resection. Furthermore, the rate of major complications has remained low over the past years, with the in-hospital mortality rate being only $0.18 \%$ 


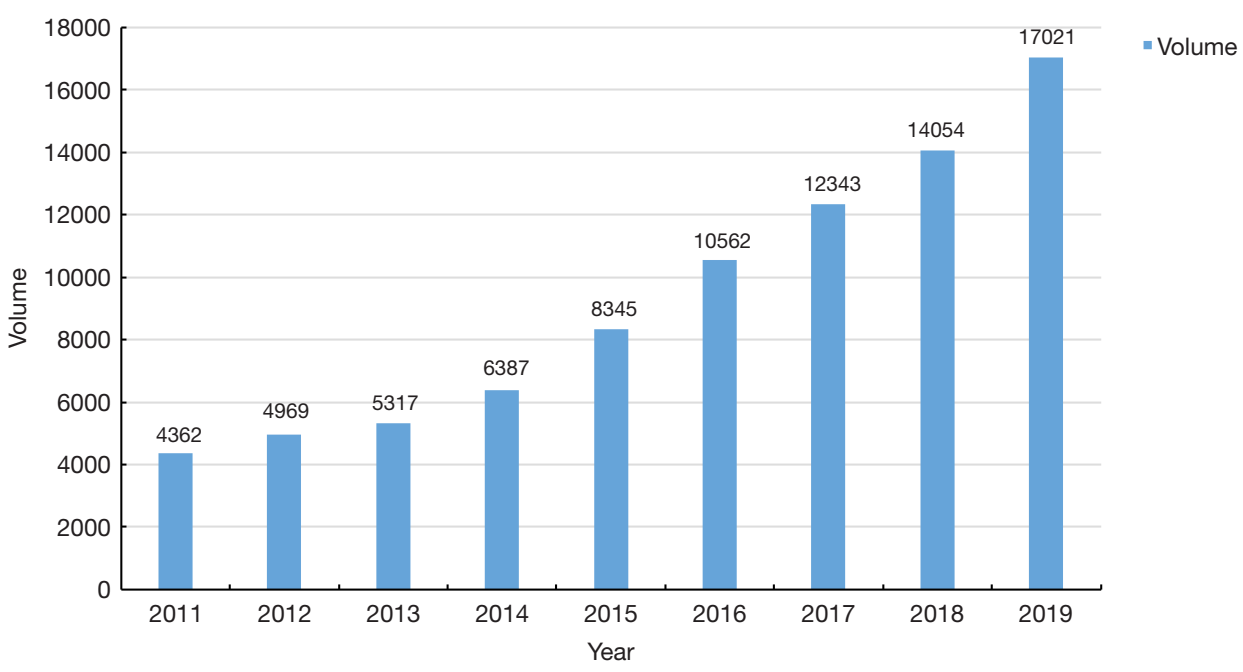

Figure 1 Case volume of thoracic surgery in Shanghai Chest Hospital.

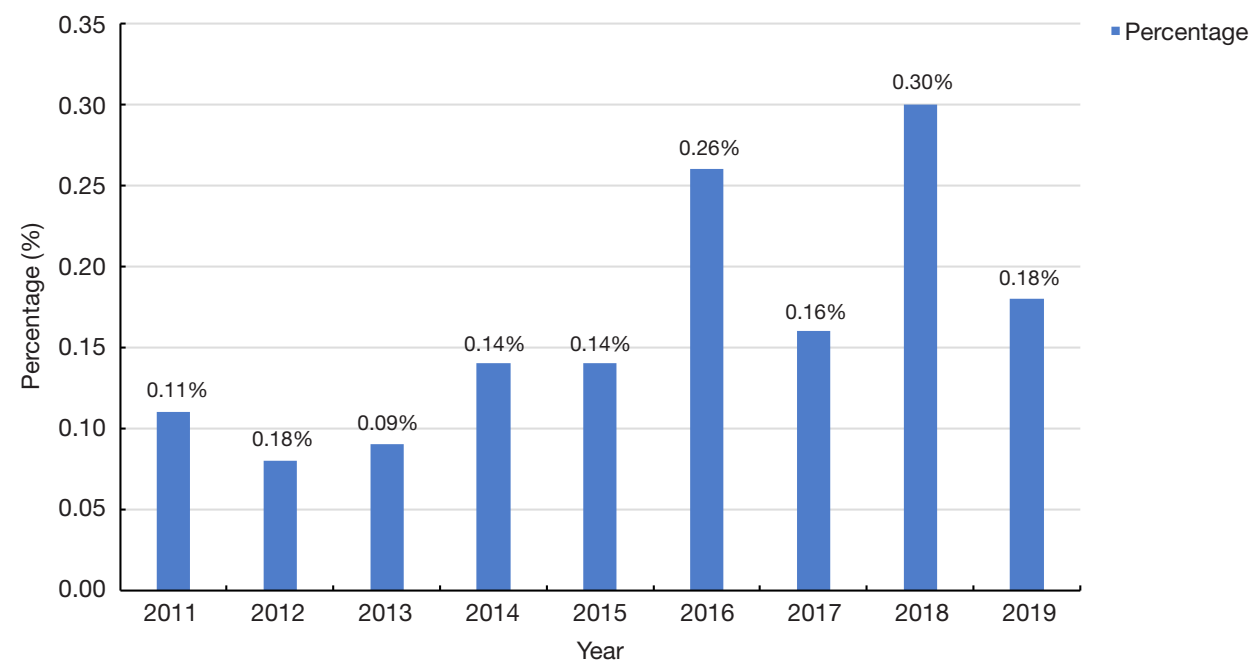

Figure 2 Mortality after thoracic surgery in Shanghai Chest Hospital.

in 2019 (Figure 2). This report presents our surgery volume, the quality of our service, and our efforts to move thoracic surgery forward so as to better help our patients.

\section{Methods}

\section{Patients}

The data of patients receiving thoracic surgeries at the Department of Thoracic Surgery and the Department of Oncological Surgery of Shanghai Chest Hospital from January 2019 to December 2019 were retrospectively collected. These patients were classified into pulmonary, esophageal, mediastinal, tracheal, and lung transplantation divisions according to different diseases and surgical treatments they received. Additionally, data on the volume of surgical resections, the surgical types and approaches, the pathological subtypes, the perioperative outcomes, and other characteristics were collected.

\section{Statistical analysis}

Categorical and continuous variables are presented as frequencies (percentages) and mean \pm standard deviation 


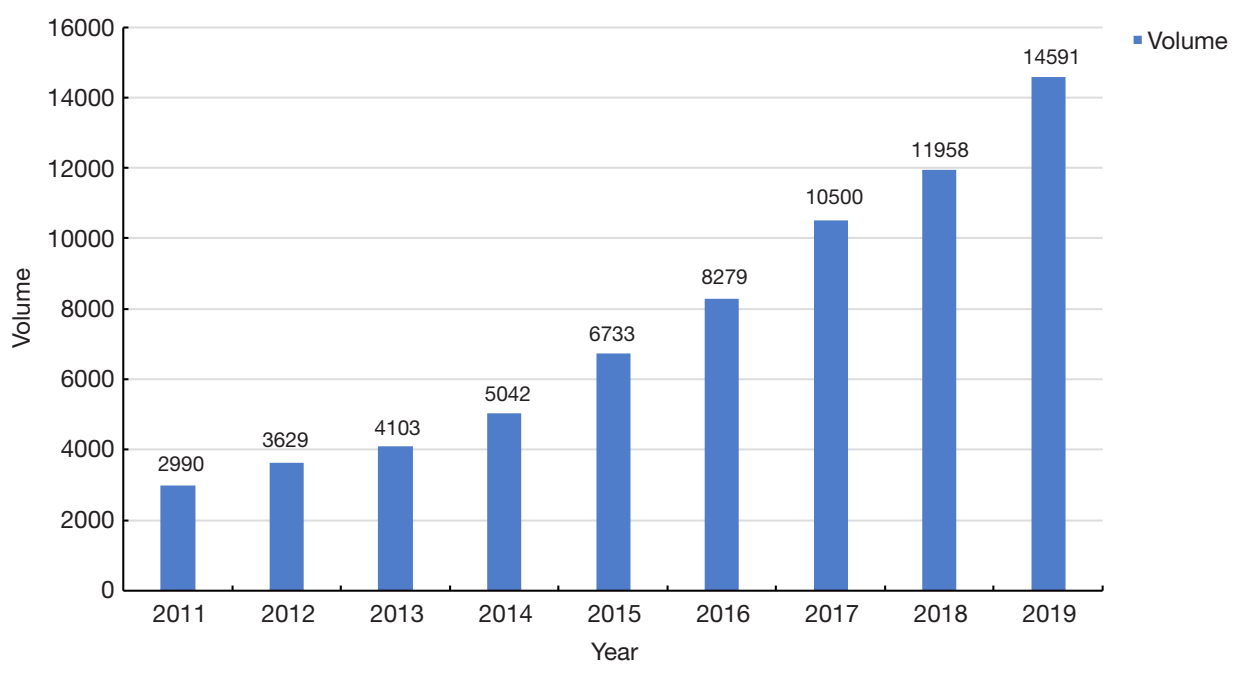

Figure 3 Total number of lung operations in Shanghai Chest Hospital.

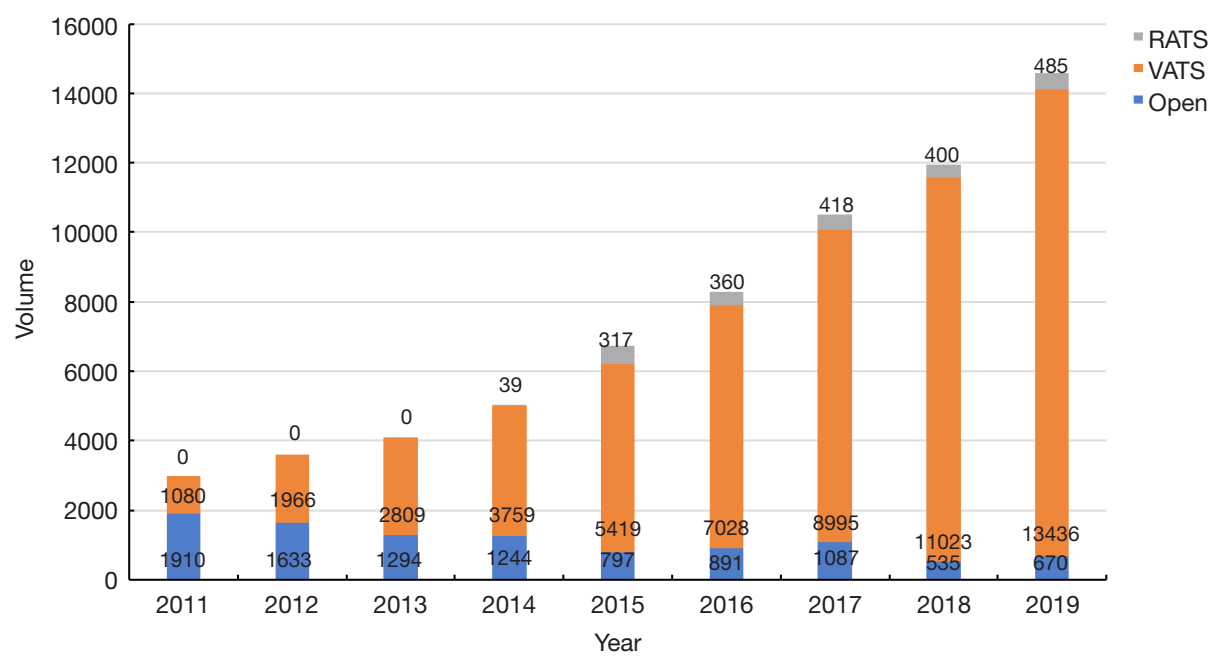

Figure 4 Pulmonary surgery approaches in Shanghai Chest Hospital.

(SD). Bar, line, and pie graphs were plotted to visually represent the information. Statistical analysis was performed using SPSS v. 22.0 (IBM Corp., Armonk, NY, USA).

\section{Results}

\section{Pulmonary surgeries}

In 2019, a total of 14,591 patients with pulmonary diseases underwent surgical resections at the Shanghai Chest Hospital. This number represents a $22.0 \%$ increase from the year prior (Figure 3). Among these patients, 13,436 received VATS resection (92.1\%), 485 patients received RATS resection $(3.3 \%)$, and 670 patients received open thoracotomy (4.6\%) (Figure 4).

Of the 14,591 pulmonary patients, $12,886(88.3 \%)$ were confirmed as having malignant diseases and 1,705 cases $(11.7 \%)$ had benign diseases. The rate of malignancy among all pulmonary patients was as high as $88.3 \%$. The most common surgical procedure was lobectomy $(\mathrm{n}=7,626$, $63.0 \%)$, followed by wedge resection $(n=3,968,27.2 \%)$, segmentectomy $(\mathrm{n}=1,154,7.9 \%)$, pneumonectomy $(\mathrm{n}=103$, $0.7 \%)$, sleeve resection $(\mathrm{n}=89,0.6 \%)$, Pancoast tumor resection $(\mathrm{n}=4,0.02 \%)$, and others $(\mathrm{n}=75,0.5 \%)$ (Figure 5$)$. 


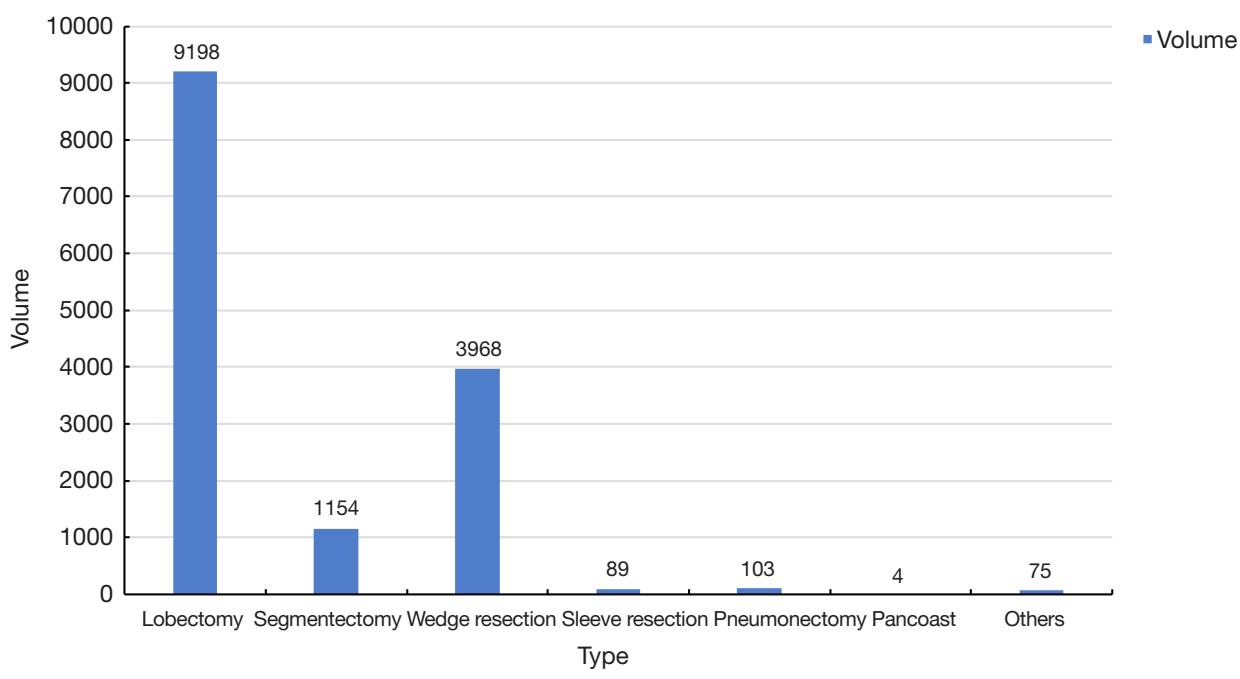

Figure 5 Surgical types for lung diseases in Shanghai Chest Hospital 2019.

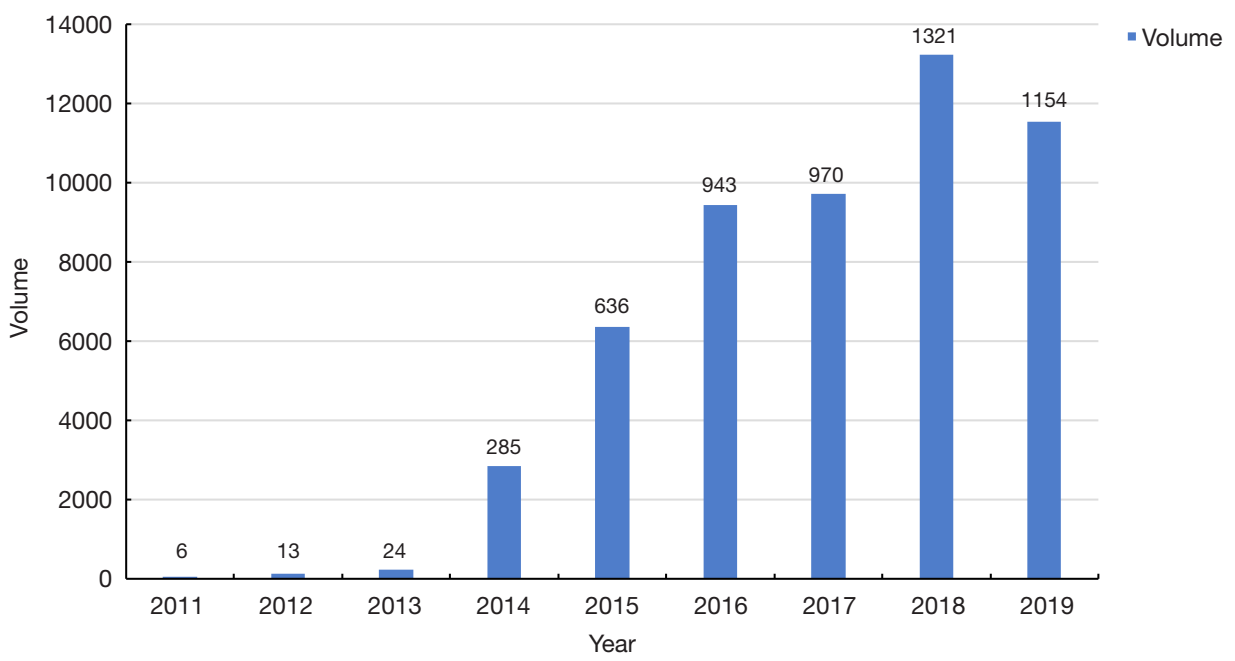

Figure 6 Number of segmentectomy in Shanghai Chest Hospital.

The volume of sublobar resections has been continuingly increasing over the past few years, owing to the increase in screening-detected small, early-stage lung cancers, especially those appearing as ground-glass opacities (GGO) on computed tomography (CT) scans. In 2019, the number of segmentectomies exceeded 1,100 cases, almost all of which were completed via minimally invasive approaches (Figure 6).

The thoracic surgery team at the Shanghai Chest Hospital has long been known for its expertise in complex pulmonary surgeries including Pancoast tumor resection, extended lobectomy, resection of stage T4 lung cancer, extrapleural pneumonectomy, etc. In addition to surgery for early-staged lung cancers, MIS also constituted a significant part of complicated pulmonary procedures for locally advanced disease. Among a total of 89 sleeve resections in 2019,8 cases $(9.0 \%)$ were successfully accomplished via MIS. In addition, VATS was successfully performed in $34(33.0 \%)$ cases of the 103 pneumonectomies, 2 were conducted by RATS resection. Excellent perioperative results in these cases indicated that MIS is safe and feasible for complicated procedures, and more patients can be expected to benefit from similar advances in surgical techniques in the future. 


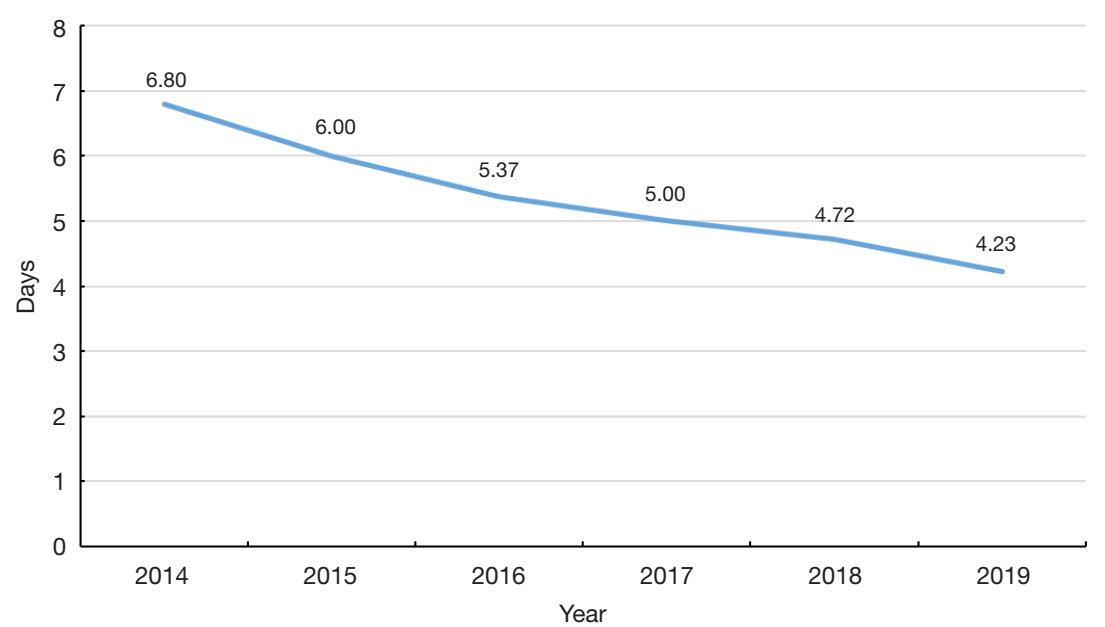

Figure 7 Average length of hospital stay after pulmonary surgery in Shanghai Chest Hospital.

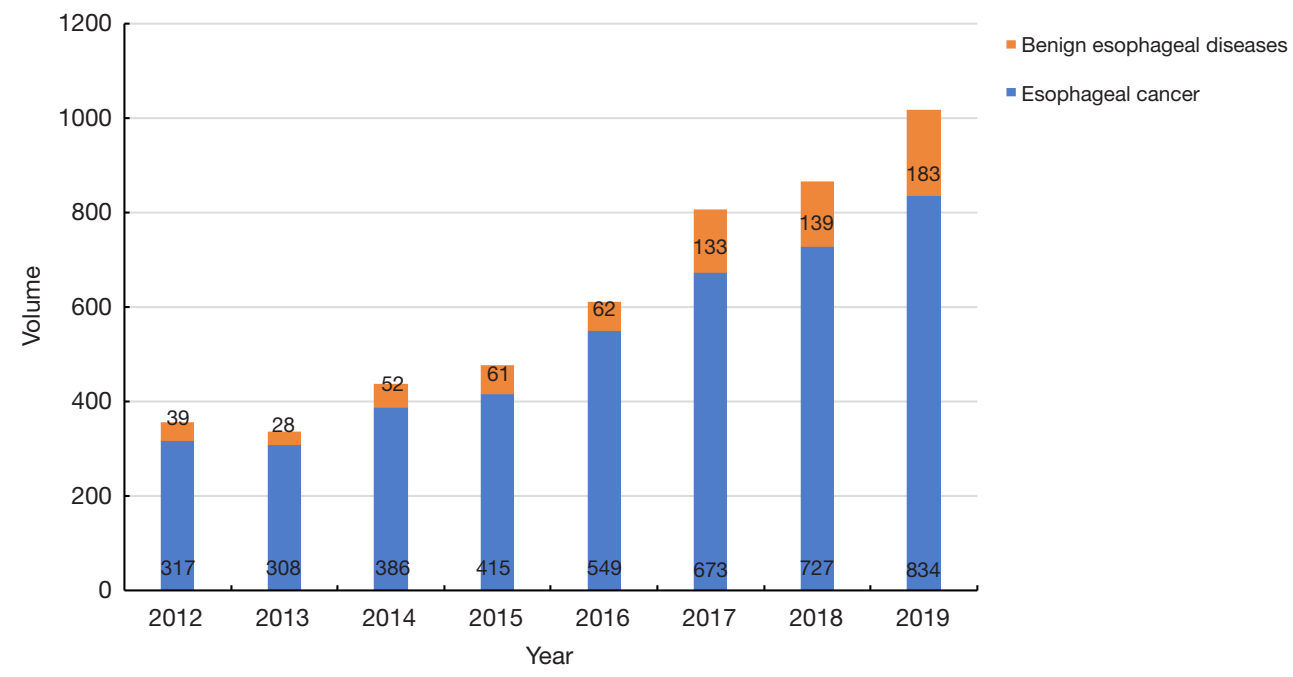

Figure 8 Case volume of esophageal surgeries in Shanghai Chest Hospital.

For the postoperative outcomes, the average length of hospital stays (LOS) after surgery was 4.23 days, and thus LOS has been successively reduced in each of the past 6 years as a consequence of the wider application of enhanced recovery after surgery (ERAS) concepts (Figure 7). The rate of major postoperative complications for pulmonary surgeries was only $9.2 \%$, with in-hospital mortality being $0.048 \%$.

In summary, the thoracic surgery team at Shanghai Chest Hospital made significant progress both in service volume and quality of pulmonary surgery in 2019. This is evidenced by the shortened LOS via the application of ERAS, and the increased use of MIS for complicated lung cancer surgeries in the treatment of locally advanced diseases.

\section{Esophageal surgeries}

The year 2019 was the first to see the total number of esophageal procedures exceed 1,000, reaching a historical height of 1,017. A total of 834 esophageal cancer patients and 183 patients with benign esophageal diseases received surgical treatment at the Shanghai Chest Hospital (Figure 8). Among the 748 esophagectomies, only 131 (17.5\%) were open cases, while minimally invasive esophagectomies (MIE) accounted for the other $82.5 \%$ cases, including 484 (64.7\%) 


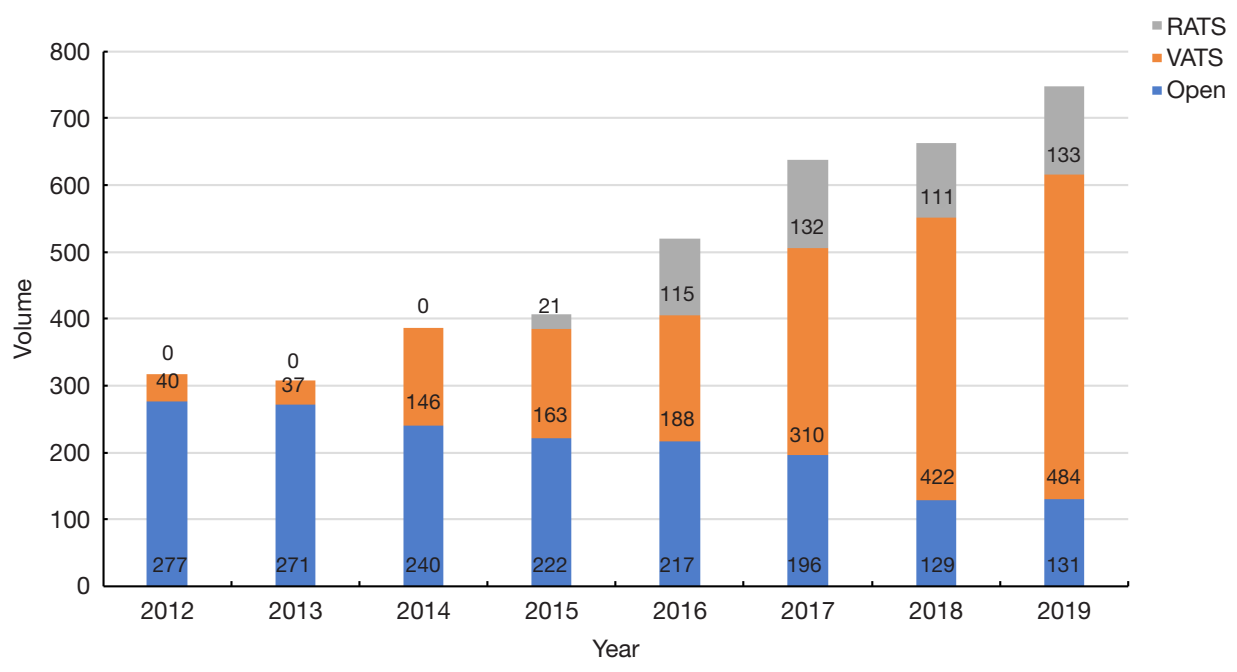

Figure 9 Surgical approaches for esophagectomy in Shanghai Chest Hospital.

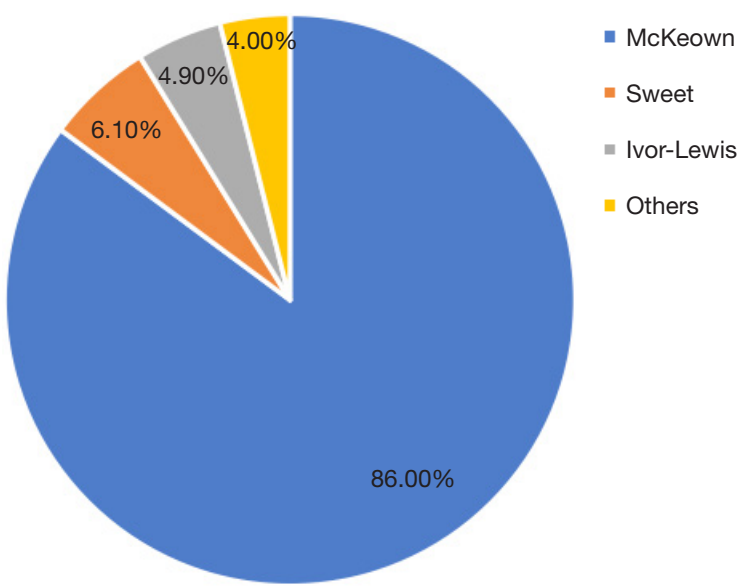

Figure 10 Surgical approaches for esophagectomy in Shanghai Chest Hospital.

video-assisted esophagectomies and 133 (17.8\%) roboticassisted esophagectomies (Figure 9).

Similar to other populations in China, the vast majority of our esophageal cancer patients had tumors located in the thoracic esophagus, with $59(7.9 \%)$ tumors in the esophageal-gastric junction (EGJ) and $24(3.2 \%)$ in the cervical esophagus. The most common histology was squamous cell carcinoma $(680,90.9 \%)$, followed by adenocarcinoma $(54,7.2 \%)$, neuroendocrine carcinoma $(12,1.6 \%)$, and other types of malignant tumors (2, $0.3 \%)$. Accordingly, McKeown is still the most preferred procedure, being applied in $643(86.0 \%)$ patients, followed by left-thoracic Sweet procedure in $46(6.1 \%)$ patients,
Ivor-Lewis procedure in 29 (4.9\%) patients, and laryngopharyngo-esophagectomy or transhiatal esophagectomy in another $30(4.0 \%)$ patients with cervical or EGJ lesions (Figure 10).

The 30 -day mortality rate was a mere $0.4 \%$, with a mean length of postoperative hospital stay of 12.58 days, representing a reduced LOS compared with the previous 6 years as consequence of ERAS adherence (Figure 11). The postoperative complication rate was $36.4 \%$, and 27 (3.6\%) patients experienced Clavien-Dindo grade III-IV complications.

Endoscopic submucosal dissection (ESD) has been increasingly used in patients with early (mucosal and superficial submucosal) esophageal cancers. Ninetythree cases of ESD were successfully completed in 2019, which represents an increase of $43.1 \%$ compared to 2018 (Figure 12). Only a single patient (1.1\%) needed to undergo conversion to esophagectomy due to perforation during ESD. The mean LOS was $3.5 \pm 2.6$ days, and no other major complications were observed. In addition, 7 patients with synchronous esophageal-hypopharyngeal cancers received ESD for their superficial hypopharyngeal lesion in combination with esophagectomy for esophageal cancer.

Notably, the number of patients with benign esophageal diseases increased by $31.7 \%$ compared to 2018 . Among the 183 cases of benign esophageal disease, 39 (21.3\%) had benign esophageal tumors, $14(7.7 \%)$ had gastroesophageal reflux disease, 25 (13.7\%) had hiatus hernias, 8 (4.4\%) had diverticula, 8 (4.4\%) had achalasia, 13 (7.1\%) had trachealesophageal fistulae, 20 (10.9\%) had benign esophageal 


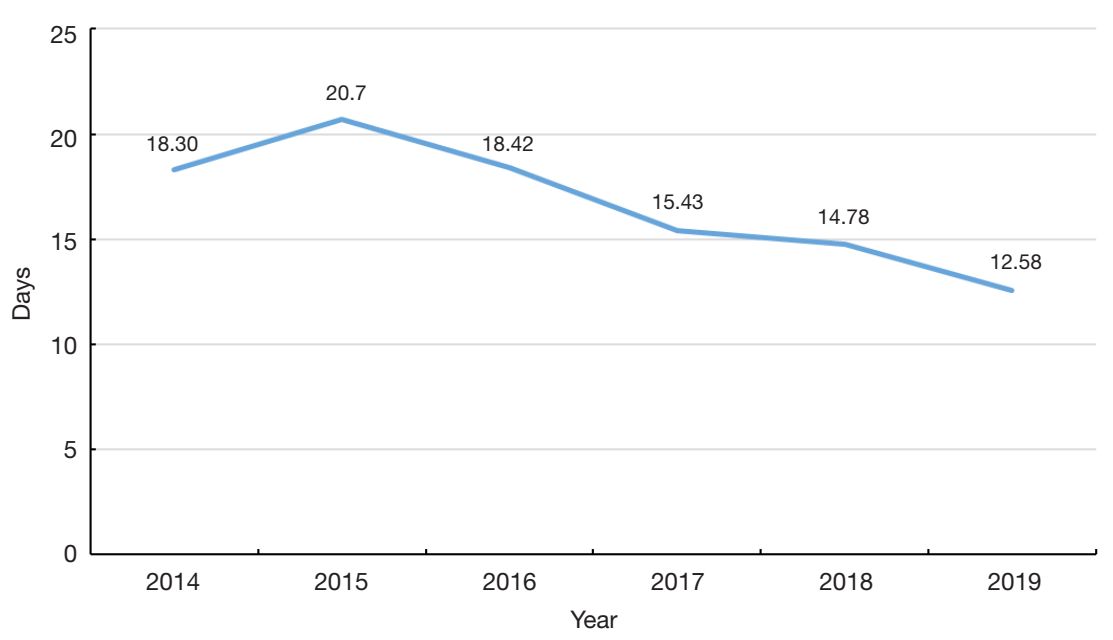

Figure 11 Average length of hospital stay after esophageal surgery in Shanghai Chest Hospital.

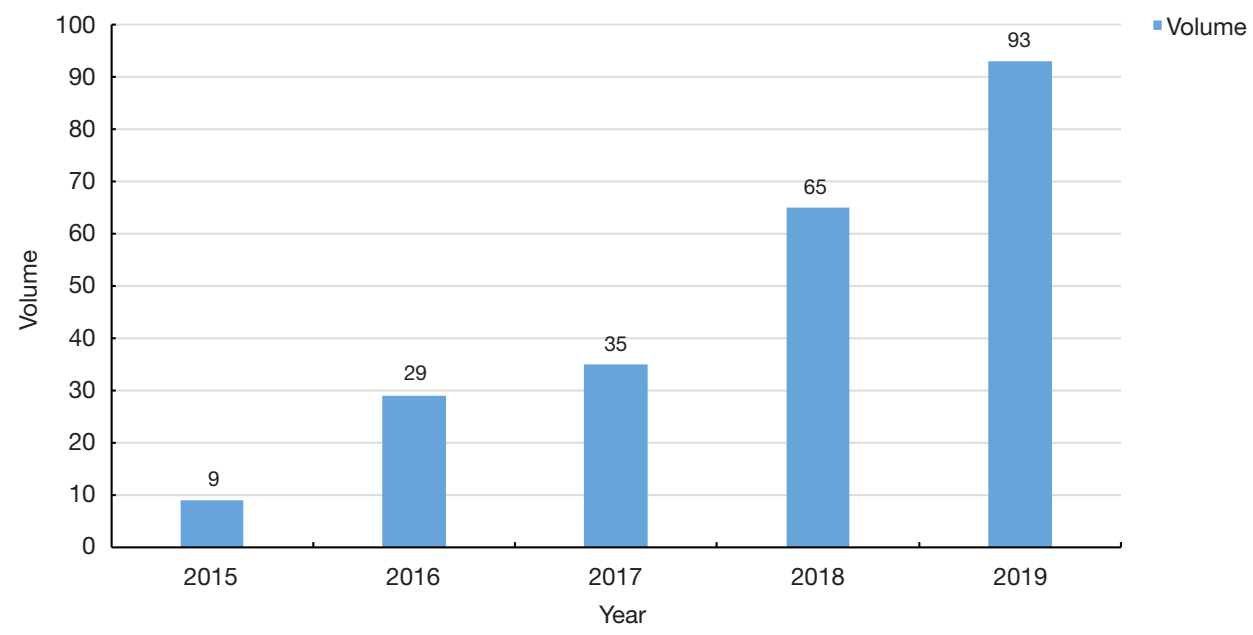

Figure 12 Case volume of endoscopic submucosal dissection for esophageal cancer in Shanghai Chest Hospital.

stenoses, and $56(30.6 \%)$ had other pathologies. Again, most of benign esophageal diseases were treated via minimally invasive approaches, including 58 (31.7\%) endoscopic therapies, $61(33.3 \%)$ thoracoscopic/laparoscopic-assisted surgeries, and $6(3.3 \%)$ robotic-assisted surgeries.

Over the past year, the esophageal surgery division at the Shanghai Chest Hospital has constantly improved the minimally invasive techniques and endoscopic therapies for malignant and benign esophageal diseases. In the meantime, complex procedures like colonic and freejejunum interposition for esophageal substitution, total laryngo-pharyngo-esophagectomy, and others, have also been attended to with persistent effort, particularly when treating difficult cases.

\section{Mediastinal surgeries}

The total case volume of mediastinal procedures was also remarkable, and reached a historical peak of 1,089 in 2019. Again, MIS was the main approach, with 691 VATS procedures $(63.5 \%)$ and 89 RATS procedures $(8.2 \%)$. Open thoracotomy or median sternotomy was still needed in 309 patients, and thus $28.3 \%$ of our cases were challenging procedures for locally advanced diseases (Figure 13). Consistent with the increasing number of complicated surgeries (Figure 14), 2019 saw more patients 


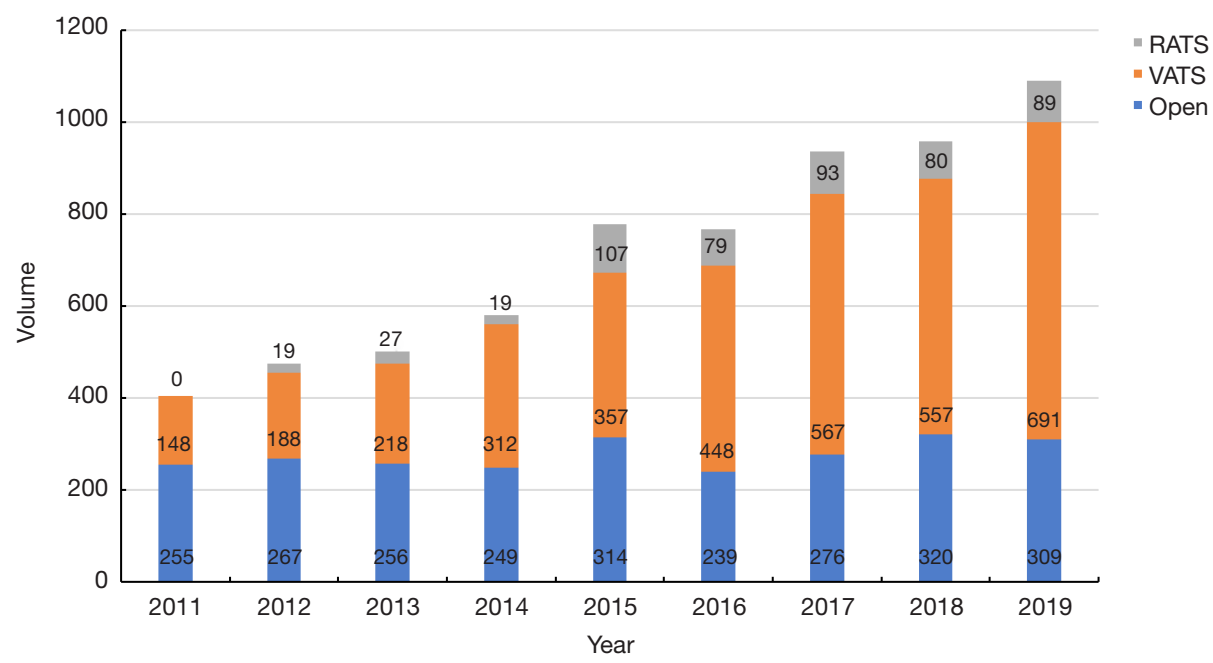

Figure 13 Mediastinal surgical approaches in Shanghai Chest Hospital.

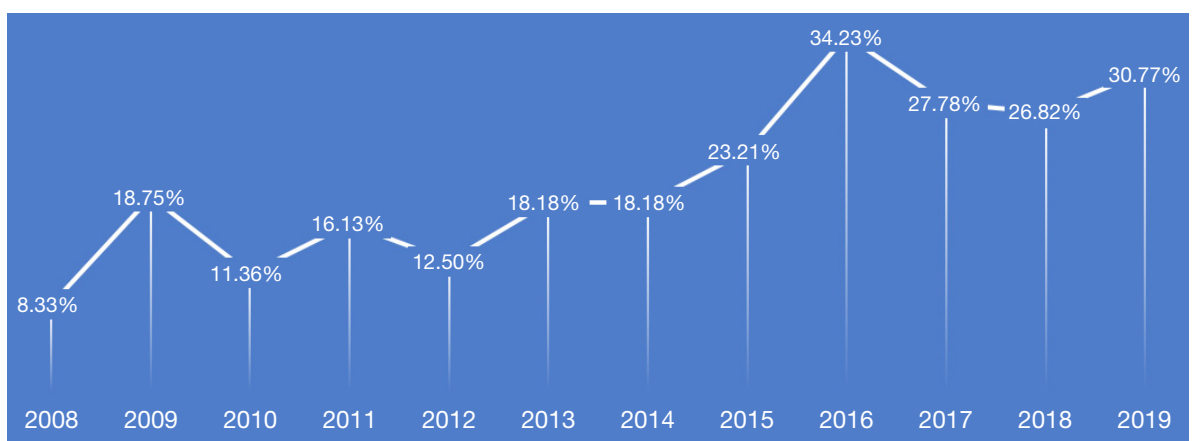

Figure 14 Percentages of preoperative therapy for locally advanced thymic tumor patient seach year.

receiving preoperative induction therapies (56 cases, $30.8 \%$ of stage III-IV patients) than the year before.

Among the 1,089 patients, 538 had thymic epithelial tumors (TET, including 35 cases with recurrent diseases), 254 had mediastinal cysts, 89 had neurogenic tumors, 75 had germ cell tumors, and 27 had hematopoietic tumors (Figure 15). Among the 503 primary TETs, 377 were thymomas (25 type A, 134 type AB, 203 type B, 15 other thymomas), 106 were thymic carcinomas, and 20 were neuroendocrine thymic tumors (NETT) (Figure 16). Of the 35 recurrent cases, $91 \%$ were type B thymomas presenting as pleural dissemination. Among the 75 germ cell tumors, 47 were teratomas, 5 were seminomas, 7 were yolk sac tumors, and 16 were mixed type non-seminomatous germ cell tumors.

Importantly, the number of complicated anterior mediastinum surgeries performed with VATS for
TETs [TNM staging II-III according to the Union for International Cancer Control (UICC) 8th edition] continued to increase in 2019 (Figure 17). In addition, the number of complicated anterior mediastinum surgeries by open procedures for TETs (UICC $8^{\text {th }}$ TNM staging II-IV) decreased when compared to last year, with open procedures being performed in 108 patients in 2019 (35.0\% of all open surgeries) (Figure 18).

Overall, only $21(1.93 \%)$ patients suffered major post-operative complications, with $2(0.18 \%)$ patients succumbing during the postoperative period. With the help of ERAS, the post-operative LOS decreased from 5.8 days in 2018 to 4.95 days in 2019. These results are extremely promising, especially in simple and straightforward minimally invasive mediastinal surgeries. No severe complications or readmissions were needed for ERAStreated patients in 2019 . 


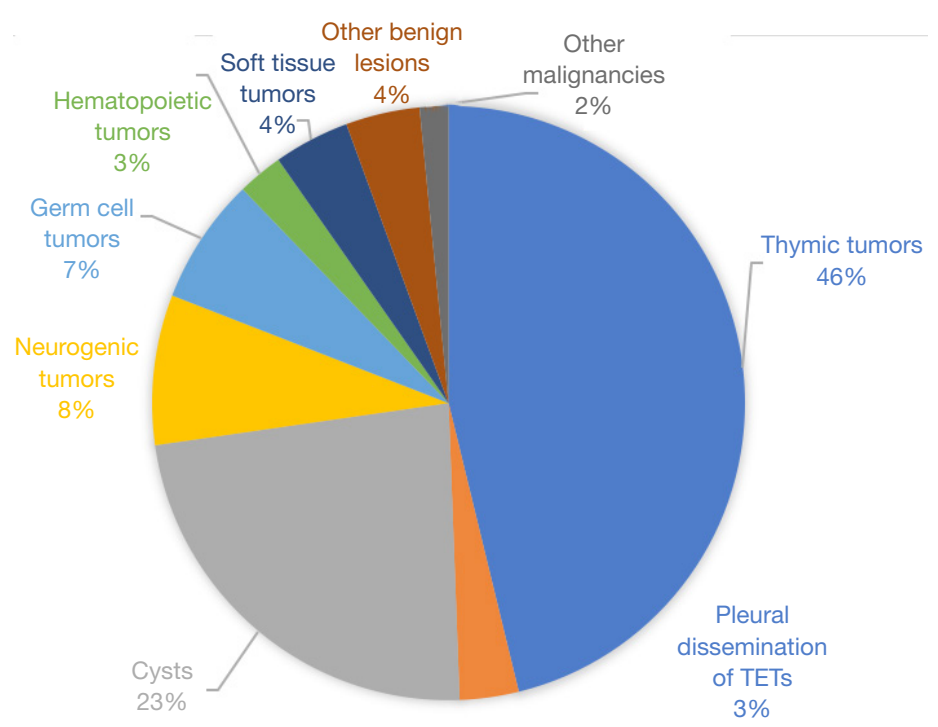

Figure 15 Mediastinal tumor histologies in 2019.

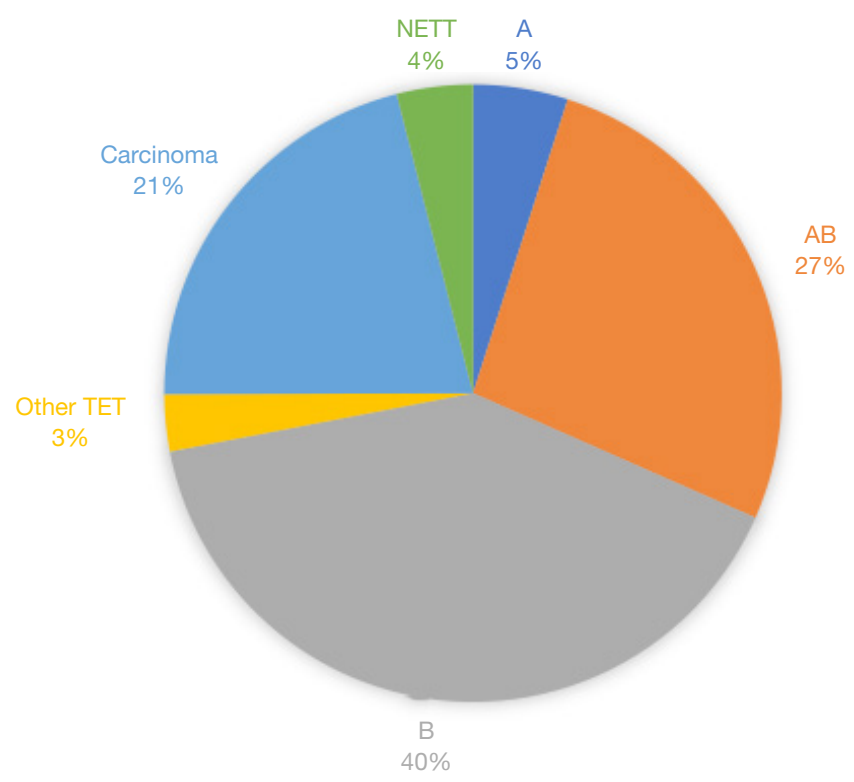

Figure 16 Subtypes of thymic malignancies.

The mediastinal surgery division of Shanghai Chest Hospital has continually pushed the application of MIS in the surgical treatment of mediastinal diseases. This is evidenced by the high percentage of RATS and VATS performed for locally invasive lesions as well as for early-stage tumors, and by the implementation of novel techniques such as subxiphoid VATS, uniportal
VATS, bilateral VATS, and others. Meanwhile, complex mediastinal surgeries for extensive resections of locally advanced diseases have not been compromised. Intentional and salvage surgeries after neoadjuvant therapies, resection and reconstruction of great vessels, and re-resection and pleurectomy for recurrent diseases were routinely carried out on a daily base, representing our unrelenting effort in 


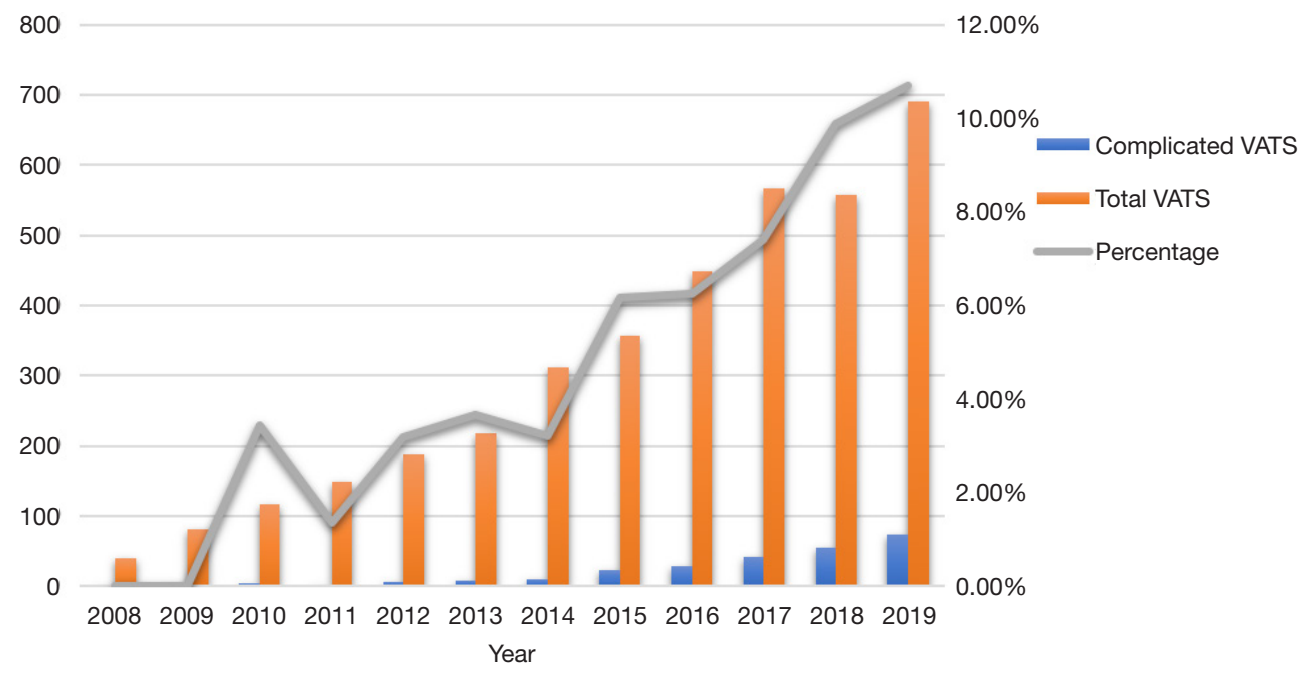

Figure 17 Complicated anterior mediastinum surgery by VATS for TETs. VATS, video-assisted thoracic surgery; TET, thymic epithelial tumors.

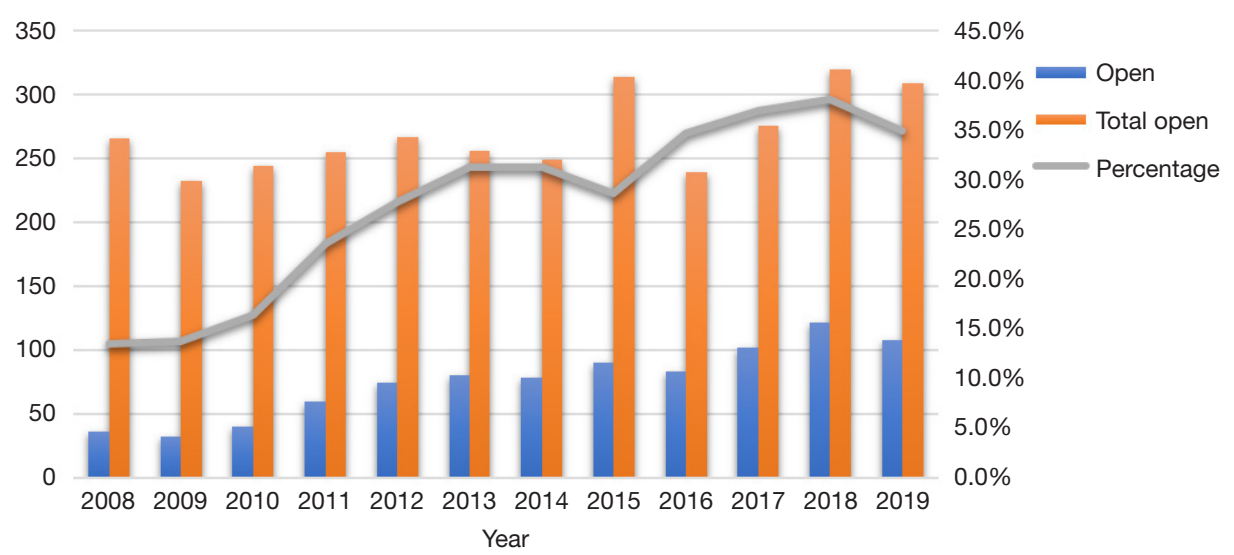

Figure 18 Complicated anterior mediastinal procedures by open surgery for TETs. TET, thymic epithelial tumors.

helping those patients with refractory diseases.

\section{Tracheal surgeries}

As one of the leading centers in tracheal surgery in China, the Shanghai Chest Hospital completed 59 tracheal procedures in 2019 , representing a $31.1 \%$ increase from 2018 (Figure 19). These included 21 cases $(37.2 \%)$ of tracheal surgeries, 22 cases $(37.2 \%)$ of main bronchial surgeries, and 9 cases (13.6) of carina resection and reconstruction (Figure 20). The average LOS was $10.5 \pm$ 8.2 days, the major postoperative complication rate was $32.2 \%$, and the rate of in-hospital mortality was $1.7 \%$.
Improvements made in complicated surgery for tracheal and bronchial diseases by our tracheal team in the year of 2019 included laryngotracheal reconstruction, VATS thoracic tracheal reconstruction under non-intubated anesthesia, VATS carina reconstruction, and others. More patients are thus expected to benefit from these advances in surgical techniques.

\section{Lung transplantations}

In 2019, 9 patients registered on the waiting list to receive lung transplantation, including 3 cases of sequential double-lung transplantation, 3 cases of left single- 


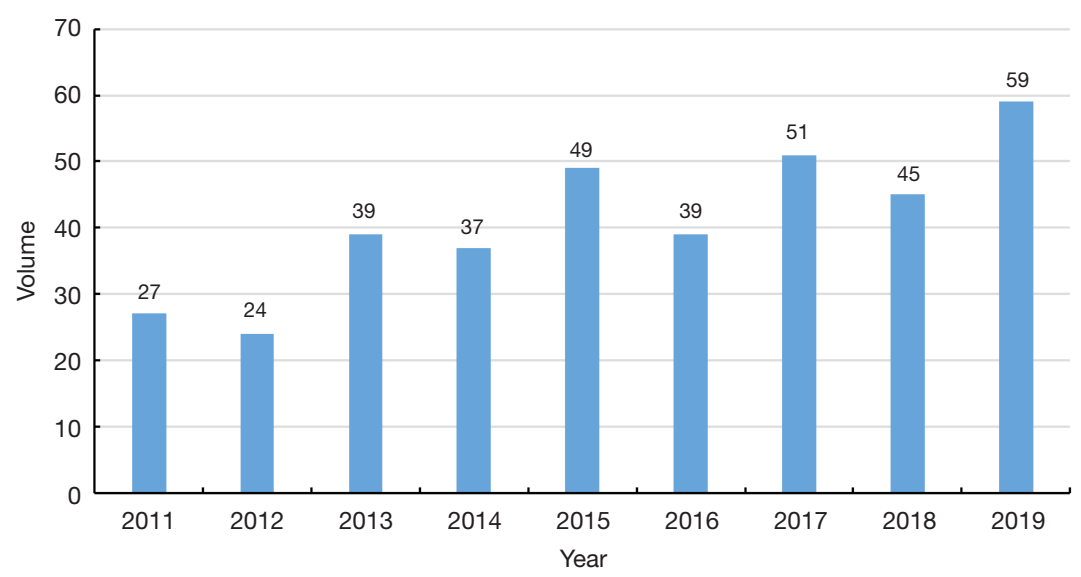

- Volume

Figure 19 Case volume of tracheal surgery in Shanghai Chest Hospital.

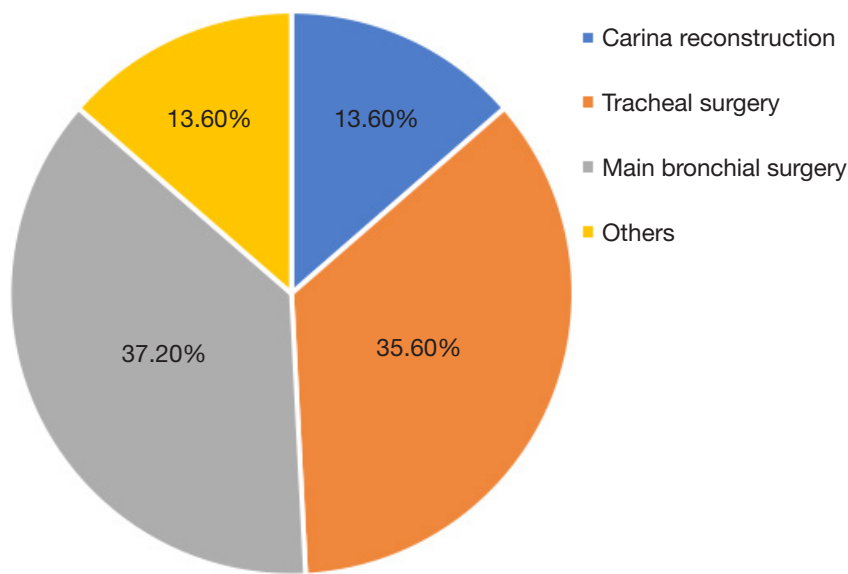

Figure 20 Surgical approaches of tracheal diseases in Shanghai Chest Hospital.

lung transplantation, and 3 cases of right single-lung transplantation. More efforts are needed to find donors, while research in improving the quality of donor organs is also underway.

\section{Discussion}

The thoracic surgery team of Shanghai Chest Hospital has been publishing our annual reports to the public since 2017 . Summarized in these reports are the major achievements made in past year, in addition to the increasing need for services at the Shanghai Chest Hospital. As one of the largest thoracic surgery centers in China, the annual volume of thoracic surgery at the Shanghai Chest Hospital has exceeded 10,000 cases for 4 consecutive years and reached a new high of more than 17,000 in 2019. Minimally invasive thoracic surgery is playing an increasingly important role in our daily practice, with nearly $90 \%$ of all procedures being completed by MIS approaches. This is in line with the rapid increase in the screening and early detection of lesions, and the rising demand for faster recovery and better quality of life from our patients. Notably, the volume of RATS has also been steadily increasing over the past years, making our hospital one the largest RATS centers in the world.

Being one of the most historic cardiothoracic centers in China, the Shanghai Chest Hospital has long been known for its ability and experience in the management of complex, difficult, and refractory chest diseases. In recent years, one of our focal areas has been the use of MIS for patients with locally advanced diseases and those needing complex procedures, including various reconstructions after resection. We are happy to see that our attempts in MIS after neoadjuvant therapies or for those patients with locally advanced diseases have been successful. In the management of lung cancer, esophageal cancer, mediastinal tumors, and tracheal diseases, similar or superior outcomes to open surgery were observed in major postoperative complication rates, in-hospital mortality rates, and rates of unplanned reoperation or readmission to ICU, while oncological results such as complete resection or lymph node dissection were not compromised. These encouraging results suggest that we have improved our service to patients by providing them state-of-the-art healthcare. We truly hope that this year's annual report from the thoracic surgery team of Shanghai Chest Hospital can again serve as a useful reference for our colleagues. We will continue to spare no effort in providing high-level medical care and dedicated 
service.

\section{Acknowledgments}

Funding: None.

\section{Footnote}

Provenance and Peer Review: This article was commissioned by the editorial office, Shanghai Chest. The article did not undergo external peer review.

Conflicts of Interest: All authors have completed the ICMJE uniform disclosure form (available at http://dx.doi. org/10.21037/shc-2021-01). QL serves as an unpaid Associate Editors-in-Chief of Shanghai Chest. WF serves as an unpaid Executive Editor-in-Chief of Shanghai Chest. The authors have no other conflicts of interest to

doi: 10.21037/shc-2021-01

Cite this article as: Wang Y, Gu Z, Yao F, Mao T, Wang R, Sun Y, Li Z, Yang J, Tan Q, Luo Q, Fang W. Annual report of thoracic surgery service at Shanghai Chest Hospital in 2019. Shanghai Chest 2021;5:13. declare.

Ethical Statement: The authors are accountable for all aspects of the work in ensuring that questions related to the accuracy or integrity of any part of the work are appropriately investigated and resolved.

Open Access Statement: This is an Open Access article distributed in accordance with the Creative Commons Attribution-NonCommercial-NoDerivs 4.0 International License (CC BY-NC-ND 4.0), which permits the noncommercial replication and distribution of the article with the strict proviso that no changes or edits are made and the original work is properly cited (including links to both the formal publication through the relevant DOI and the license). See: https://creativecommons.org/licenses/by-nc-nd/4.0/.

(English Language Editor: J. Gray) 